\title{
INNER FUNCTIONS ON THE POLYDISC ${ }^{1}$
}

\author{
S. H. KON
}

\begin{abstract}
It is shown that the inner functions on the polydisc, unlike the classical case of the unit disc, fail to separate the points of the maximal ideal space of $H^{\infty}\left(U^{n}\right)$. From this we deduce that the inner functions generate a proper closed subalgebra of $H^{\infty}\left(U^{n}\right)$.
\end{abstract}

1. Introduction. Let $T^{n}$ be the distinguished boundary of the unit polydisc $U^{n}$. The class of all bounded analytic functions on $U^{n}$ is denoted by $H^{\infty}\left(U^{n}\right)$. It is well known that for $f \in H^{\infty}\left(U^{n}\right)$, the radial limit

$$
f^{*}(\omega)=\lim _{r \rightarrow 1} f(r \omega)
$$

exists for almost every $\omega \in T^{n}$ with respect to the normalised Lebesgue measure. A bounded analytic function is said to be an inner function if its radial boundary function is of absolute value one almost everywhere on $T^{n}$.

Let $J\left(U^{n}\right)$ denote the closed subalgebra generated by the inner functions in $H^{\infty}\left(U^{n}\right)$. Identifying a bounded analytic function with its radial boundary function $f^{*}, H^{\infty}\left(U^{n}\right)$ and $J\left(U^{n}\right)$ can be identified with closed subalgebras of $L^{\infty}\left(T^{n}\right)$.

With pointwise addition and multiplication, $H^{\infty}\left(U^{n}\right)$ and $L^{\infty}\left(T^{n}\right)$ are Banach algebras under the supremum norm. Let $M_{n}$ and $X_{n}$ be the maximal ideal space of $H^{\infty}\left(U^{n}\right)$ and $L^{\infty}\left(T^{n}\right)$ respectively. Define $\tau: X_{n} \rightarrow M_{n}$ by mapping each complex homomorphism of $L^{\infty}\left(T^{n}\right)$ to its restriction on $H^{\infty}\left(U^{n}\right)$. The Shilov boundary of $H^{\infty}\left(U^{n}\right)$ will be denoted by $\partial_{n}$ and the Gelfand transform of an element $f$ by $\hat{f}$. For the unit disc $U$, the index $n=1$ will be omitted from our notations.

For the classical case of $H^{\infty}\left(U^{n}\right)$, it is well known that $\tau$ is a homeomorphism from $X$ into $M$ and $\partial=\tau X$, see [2]. For $n>1, \tau: X_{n} \rightarrow M_{n}$ is still continuous, but as shown in [5], it is not one-to-one and $\partial_{n} \neq \tau X_{n}$.

A result of Hoffman together with a result of Douglas and Rudin show that the inner functions separate the points of all of the maximal ideal space $M$ of $H^{\infty}(U)$, see [1].

Received by the editors December 6, 1977 and, in revised form, June 5, 1978.

AMS (MOS) subject classifications (1970). Primary 32A30, 46J20, 46J30; Secondary 46J10, 46J15.

Key words and phrases. Inner functions, bounded analytic functions, polydisc, $H^{\infty}\left(U^{n}\right)$, maximal ideal space, Shilov boundary, $L^{\infty}\left(T^{n}\right)$, separate points.

${ }^{1}$ This research was done under the supervision of Dr. P. S. Chee at the University of Malaya and formed part of a thesis submitted for examination for the $\mathrm{Ph}$. $\mathrm{D}$. degree. 
Recently, Marshall [4] has shown that the inner functions generate $H^{\infty}(U)$. From this it also follows that the inner functions separate the points of $M$.

Naturally the following two questions also arises for the polydisc.

(i) Do the inner functions separate the points of the maximal ideal space $M_{n}$ for $n>1$ ?

(ii) Do the inner functions generate $H^{\infty}\left(U^{n}\right)$ for $n>1$ ?

We will answer both questions in the negative in this paper.

2. Separating points of the maximal ideal space. By considering inner functions of the form $\left(z_{i}-\alpha_{i}\right) /\left(1-\bar{\alpha}_{i} z_{i}\right)$, for $\alpha_{i} \in U$, it is easy to see that the inner functions separate points of $M_{n}$ from different fibers. Using an argument of Douglas and Rudin [1], and the fact that $\partial_{n} \neq \tau X_{n}$ for $n>1$, it will be shown that the inner functions cannot separate the points of $M_{n}$. More precisely, we have the following theorem.

THEOREM 1. The Gelfand transforms of the inner functions do not separate the points of the maximal ideal space $M_{n}$ of $H^{\infty}\left(U^{n}\right)$ for $n>1$.

Proof. Suppose that the Gelfand transforms of the inner functions separate the points of $\tau X_{n}$. Let $E$ be a proper compact subset of $\tau X_{n}$, and let $\Phi_{1} \in \tau X_{n}-E$. Now for each $\Phi_{\alpha} \in E$, there exists an inner function $u_{\alpha}$ such that

$$
\hat{u}_{\alpha}\left(\Phi_{1}\right) \neq \hat{u}_{\alpha}\left(\Phi_{\alpha}\right)
$$

by our assumption. Observe that $u \in H^{\infty}\left(U^{n}\right)$ is inner if and only if $|\hat{u}(\Phi)|=$ 1 for every $\Phi \in \tau X_{n}$, see [3]. Hence without loss of generality, it can be assumed that $\hat{u}_{\alpha}\left(\Phi_{1}\right)=1$ and $\operatorname{Re} \hat{u}_{\alpha}\left(\Phi_{\alpha}\right)<1$. There is then, a neighbourhood $N\left(\Phi_{\alpha}\right)$ of $\Phi_{\alpha}$ such that

$$
\operatorname{Re} \hat{u}_{\alpha}(\Phi)<1 \text { for all } \Phi \in N\left(\Phi_{\alpha}\right) \text {. }
$$

By the compactness of $E$, there exists finitely many inner functions, say $u_{1}, \ldots, u_{m}$ such that

$$
\hat{u}_{k}\left(\Phi_{1}\right)=1 \text { for } 1 \leqslant k \leqslant m
$$

and

$$
\inf _{k} \operatorname{Re} \hat{u}_{k}(\Phi)<1 \quad \text { for every } \Phi \in E .
$$

Then $f=1+u_{1}+\cdots+u_{m} \in H^{\infty}\left(U^{n}\right)$ and $\hat{f}\left(\Phi_{1}\right)=m+1=\|\hat{f}\|$, but $|\hat{f}(\Phi)|<m+1$ for every $\Phi \in E$. Thus $E$ cannot contain $\partial_{n}$ and we deduce that $\partial_{n}=\tau X_{n}$, contradicting the result of [5]! This shows that the inner functions cannot separate the points of $\tau X_{n}$ and hence those of $M_{n}$.

Now if we assumed $J\left(U^{n}\right)=H^{\infty}\left(U^{n}\right)$, then the inner functions being generators of the commutative Banach algebra $H^{\infty}\left(U^{n}\right)$ must obviously separate points of the maximal ideal space $M_{n}$. For $n>1$, this contradicts Theorem 1 and we have the following result. 
THEOREM 2. Let $J\left(U^{n}\right)$ be the closed subalgebra generated by the inner functions in $H^{\infty}\left(U^{n}\right)$. Then

$$
J\left(U^{n}\right) \neq H^{\infty}\left(U^{n}\right) \text { for } n>1 .
$$

\section{REFERENCES}

1. R. G. Douglas and W. Rudin, Approximation by inner functions, Pacific J. Math. 31 (1969), 313-320.

2. K. Hoffman, Banach spaces of analytic functions, Prentice-Hall, Englewood Cliffs, N. J., 1962.

3. S. H. Kon, Inner functions and the maximal ideal space of $L^{\infty}\left(T^{n}\right)$, J. Austral. Math. Soc. (to appear).

4. D. E. Marshall, Blaschke products generate $H^{\infty}$, Bull. Amer. Math. Soc. 82 (1976), 494-496.

5. M. Range, A small boundary for $H^{\infty}$ on the polydisc, Proc. Amer. Math. Soc. 32 (1972), 253-255.

Department of Mathematics, University of Malaya, Kuala Lumpur 22-11, Malaysia 